\title{
Applying Tire Pressure Monitoring Devices for Traffic Management Purposes
}

\author{
Milos Krstic, Nemanja Savic, Rolf Kraemer \\ IHP \\ Im Technologiepark 25 \\ 15236 Frankfurt (Oder), Germany \\ \{krstic, kraemer\}@ihp-microelectronics.com
}

\author{
Marek Junghans \\ Institute of Transportation Systems \\ Deutsches Zentrum für Luft- und Raumfahrt e.V. (DLR) \\ Berlin, Germany \\ marek.junghans@dlr.de
}

\begin{abstract}
In this paper we perform the analysis of the popular TPMS (tire pressure monitoring systems) and their application for traffic management purposes. In particular, we evaluate several of the commercially available TPMS devices and analyze their architecture and communication features. Furthermore, we propose the architecture of an external sensor device used for effective eavesdropping of TPMS ID data. Finally, we evaluate the possibility of utilizing such systems for the identification and re-identification of traffic participants using the unique ID of TPMS sensors.
\end{abstract}

Keywords - TPMS; Traffic and Transportation Management; Wireless Sensors; FSK; ASK

\section{INTRODUCTION}

Today's requirements for safe, low-carbon, and energy efficient transportation can hardly be achieved without effective traffic and transportation management systems. Traffic management and transportation systems do not only monitor the traffic conditions, but actively control traffic and enable the assistance of the drivers to get efficiently from location A to location B. To achieve these goals, traffic is measured, the obtained traffic data is processed, accurate and reliable statistics (e.g. traffic states) is obtained, and finally traffic control measures are derived and applied. Additionally, traffic simulation tools, e.g. SUMO (Simulation of Urban Mobility) [18] or VISSIM [19], are utilized for simulating novel and powerful traffic control methods and a reliable traffic prognosis. Traditionally, traffic can be measured by counting vehicles at particular points. Such systems are usually based on inductive loop detectors, infrared detectors, laser scanners, or radar detectors $[5,15]$. Nowadays, there is a significant interest in the further development of the low-cost, low-power and reliable traffic monitoring. One of the main goals is to provide real-time information about the traffic conditions in particular areas of interest and to enable personalized optimal routes for the traffic participants for low-carbon, energy efficient, and time effective routing. In order to achieve that, the traffic management and monitoring system requires more detailed information than the pure counting data only. Correspondingly, the identification and re-identification of passing vehicles is nowadays required. In this manner, the journey times for significant statistics of the passing vehicles in the observed area can be generated, which could be further used for optimal traffic monitoring and control in real time [20, 21].
There are several possibilities to enable full identification of the passing vehicles. The obvious one is to use the number plate as the identifier. Every car has a unique plate-number, which should be normally well visible. The technology such as Automatic Number Plate Recognition (ANPR) is widely used for monitoring by police forces (for example speed control), in systems such as electronic toll collection, but also for traffic management [6]. ANPR uses License Plate Capture Cameras (LPCC) to recognize license plate numbers. However, this approach has also significant limitations. One of the serious concerns is the privacy protection, since ANPR systems are not anonymous and allow the direct link to individuals (i.e. car owners) through law enforcement databases. Additionally, ANPR requires line of sight (LOS) and its accuracy can be affected by weather conditions (e.g. illumination) or the dirt on the plate [1]. As a consequence in the state of the art of ANPR systems, the recognition accuracy is from $90 \%$ [3] up to $98 \%$ $[12,13,14]$. Regarding the distance, the plates could be recognized over the distance of 10 meters using today's equipment [4]. Finally, the costs of applying this method for traffic monitoring are significant, and huge amount of stationary detectors is required [7].

To overcome this, modern traffic systems may use the fact, that vehicles are more and more equipped with communication devices. Each of such devices as a part of the message utilizes some unique identifier, usually in the form of MAC address. As an example, vehicles can be equipped with RFID tags or barcodes, which are in common use in electronic toll collection systems, but also utilized for traffic monitoring [8]. Furthermore, the GSM data exchange in on-board mobile telephony [9] has been evaluated for such purposes. Finally, since Bluetooth technology is frequently used in vehicles, including passenger devices, there are methods for employing them for traffic statistic generation and calculation of journey times $[10,11]$.

During the last couple of years the use of Tire Pressure Monitoring Systems (TPMS) became very pervasive, mainly due to the legislation requesting such devices for safety reasons in the US from 2007 and in Europe from 2012 in each new car [1]. TPMS sensors use cheap technology, mainly based on transmission in $433 \mathrm{MHz}$ band, and triggering over inductive coupling in $125 \mathrm{KHz}$ band [1]. Currently, there is no standard specifying the message format and modulation type, and there 
are several proprietary solutions from different providers. However, it has been shown that the data sent over channel is usually not encrypted and each transferred message contains the specific 32-bit sensor ID [1]. This ID could be then utilized for identification purposes and for vehicle tracking. Such identifier enables 4.294.967.296 different combinations. Although it is theoretically possible, there is a very low probability to identify two sensors with the same ID in the observed region.

Some basic analysis of the TPMS sensor message format and their potential use for identification can be found in [1]. There has been pointed out that the traffic monitoring system based on TPMS could have many benefits and advantages over current traffic monitoring systems. First of all, it relies on the information not linked to the individual (sensor's ID), and thus privacy concerns are much more reduced in comparison to ANPR systems. Installing additional hardware in vehicles is not required for identification purposes (RFID tags, etc.), since TPMS sensors will be present in practically all vehicles in the next ten to twenty years. The traffic infrastructure would contain very cheap low-power sensor nodes, installed along the road where they can be powered using energy harvesting. Moreover, the ability to eavesdrop on the RF transmission of TPMS packets does not depend on illumination or LOS [1]. The probability of identifying the sensor ID is around 99\% when the eavesdropper is placed 2.5 meters away from the lane marks [1], which is better than ANPR method. Finally, the humane nature must be taken into account. The LOS requirement forces the ANPR to be installed in visible locations. Therefore, the drivers frequently choose alternative routes, or they change their usual traffic behavior (e.g. reduce the speed significantly), which affects the quality of obtained statistics [1]. Some analyses of TPMS sensors have been made in [16] and some ideas of their applications have been recently published in [17].

In the following we will thoroughly analyze the features of TPMS systems, utilized modulations and message format of some of the popular TPMS devices. Furthermore, we will investigate the architecture of the sensing devices used for eavesdropping of the ID information and other relevant data. Finally, we will analyze the scenarios and chances for applying such devices for modern traffic management systems.

\section{CONCEPT AND ARCHITECTURE OF TPMS}

A TPMS is a driver-assistance system that warns the driver when the tire pressure is below or above the prescribed limits [2]. This technique is based on real time monitoring of the tire pressure. Two methods are there utilized: (A) direct and (B) indirect. In direct TPMS, the pressure drop is calculated based on actual pressure measurements through sensors. In contrast, measurements, e.g. wheel speeds, are used in indirect TPMS [2]. The involvement of TPMS systems should contribute to improved safety, reduced fuel consumption, extended tire lifetime, decreased emissions, etc.

A. Direct TPMS involve the sensors that physically measure the pressure of each tire. The block diagram of the typical direct TPMS architecture is given in Figure 1. The wired connection to the sensor is difficult due to the rotation of the tire. Therefore, the sensors are wirelessly connected to the central unit and use this wireless channel to transmit the measured pressure and other important sensed values (temperature etc.). In order to enable continuous pressure measurement and RF transmission these direct TPMS sensors are usually equipped with the battery or use some energy harvesting mechanism. Therefore, in order to save the energy consumption, sensor activation is performed in strictly defined scenarios. The transmission of the data could be periodical, when the vehicle is above the speed of $40 \mathrm{~km} / \mathrm{h}$, during starting diagnosis when initial connection to the sensors is made, or induced by some sort of low-frequency wake-up signal [1]. This activation signal is usually a tone or modulated signal of $125 \mathrm{KHz}$ in low frequency (LF) range. Since it is very difficult to transmit signals in this range due to the size of antenna, the usual way of addressing this is the inductive coupling enabling wake-up of the sensor over the short distance. When the wakeup sequence is recognized the sensor will send the data packets. The device controlling the activation of the sensors is called electric control unit (ECU), which is able to receive the data from each sensor using separate antennas placed near to each tire. The communication protocols used here between sensors and TPM ECUs are proprietary and thus, do not belong to any existing standard. From supplier materials it can be found that TPMS data transmissions commonly use the $315 \mathrm{MHz}$ or 433 MHz bands (UHF) and ASK (Amplitude Shift Keying) or FSK (Frequency Shift Keying) modulation. Each message consists of the sensor ID (usually 32 bit), the measured pressure value, temperature, some control bits and cyclic redundancy check (CRC) checksum. The ECU is connected to the central car computer over for instance Controller-area-Network (CAN). In this way the warning sign could be shown in real time on the central dash board of the vehicle if the pressure of some tire is not within the allowed limits. Additionally, it is possible to show the pressure of each tire on demand.

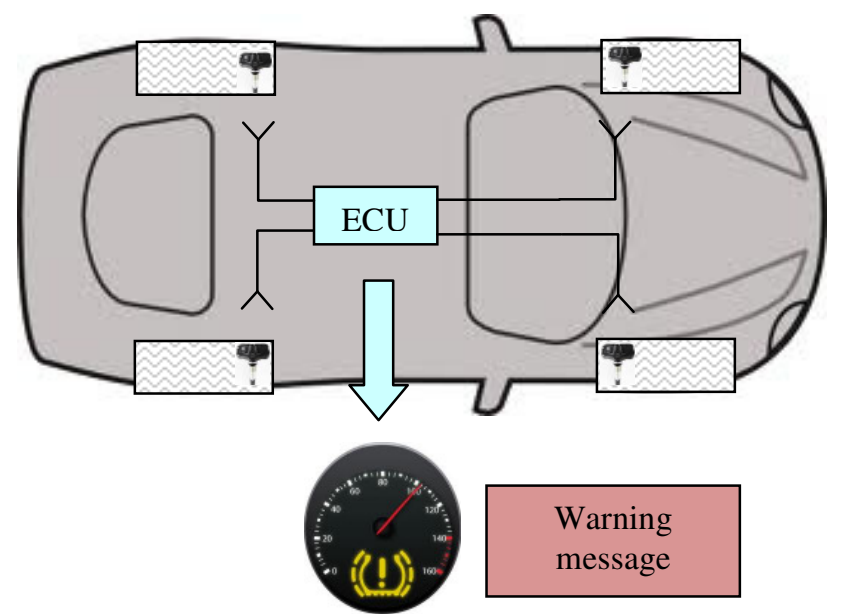

Figure 1 Architecture of the direct TPMS

B. Indirect methods are based on wheel speed measurements and require no dedicated pressure sensors. However, such a system has important deficiencies, since it does not provide the actual pressures of each tire and works only when the vehicle is in motion. Additionally, it does not warn the driver when several tires are underdeflated at the same time. Furthermore, it could indicate only when the pressure 
drop is more than $25 \%$ and, false warnings are frequent on the snowy roads [2].

Consequently, within the last years more focus is put on the direct sensors due to their application advantages. In our analysis we will also focus on the traffic management application of direct TPMS.

\section{ANALYSIS OF SELECTED TPMS DEVICES}

In order to evaluate the applicability of TPMS systems for traffic and transportation management purposes, the existing commercial TPMS sensors and their features in respect to data transmission were analyzed. TPMS manufacturers use proprietary protocols, and therefore in our analysis we put focus on three major manufacturers and corresponding sensors that are in the following text denoted as Sensor A, Sensor B, and Sensor C. The analysis of the industry relevant TPMS devices was based on their application in lab environment and corresponding triggering and measurements using the standard diagnostic tools (ATEQ VT55), spectrum analyzers, and oscilloscopes.

Since all sensors are triggered with a $125 \mathrm{KHz}$ signal, we have used parallel LC circuit tuned to that frequency in order to analyze the triggering signal over the oscilloscope. The sensors were triggered using the diagnostic tool and the response from the sensors was recorded and analyzed. Since the TPMS data exchange is not defined by the standard and each supplier uses proprietary solution we have employed reverse engineering methods. To successfully perform these tasks we took into account that each sensor must transmit its own ID as a part of the message, and this ID was already available and written on the sensor body.

To decode the message recorded data was processed to determine the modulation scheme (FSK, ASK, OOK) and encoding scheme (Manchester code, Differential Manchester code, NRZ, etc.). After performing demodulation and encoding, the ID of the sensors is easy to be extracted from the message. After that, the message format can be further disclosed. In order to determine the position of the byte containing the temperature value, several recordings with different environment temperatures were performed. The tire pressure value is extracted by recording changing pressures while the temperature was kept constant. To determine which error detection code (CRC, checksum, etc.) is used, bruteforce method was applied employing the standard polynomials.

We divided process of decoding messages in two phases:

- Recording baseband signal and storing samples in files.

- Processing recorded signals using MATLAB and extracting information (ID, temperature and pressure).

We recorded a number of messages with different pressure and temperature values. The raw baseband signal was recorded in two ways: by using USRP (Universal Software Radio Peripheral) and with a digital oscilloscope and a simple antenna. When all recordings were finished, we have started the processing using software written in MATLAB (second phase). At the first place the individual packets were extracted to speed-up the processing. Afterwards, the individual messages were bandpass filtered and demodulated. Since the sensors normally use ASK or FSK modulation, we had to implement in parallel both types of demodulators. The digital data was derived from demodulator output using Schmitt trigger that we also implemented in software. Next, the bitslicer was implemented, so that the binary bitstream is obtained. Messages are finally decoded and the information is extracted. In the following text the analysis of the sensors A, $\mathrm{B}$, and $\mathrm{C}$ is presented:

\section{Sensor A}

The analysis has shown that Sensor A is not responding to LF wake-up signal, and that data is simply periodically sent every 55 sec. The applied modulation type was identified as FSK. The spectrum of the received signal is shown in Figure 2.

Sensor A sends only one message with a duration of $16 \mathrm{~ms}$, which consists of a preamble and 10 data bytes. The preamble is used to adjust bit decision circuitry to a proper decision level and it consists of eight pulses of one half of bit rate width. The start of frame is binary value 10 (decoded) and it is followed by ten bytes of data. The applied encoding scheme is Differential Manchester code.

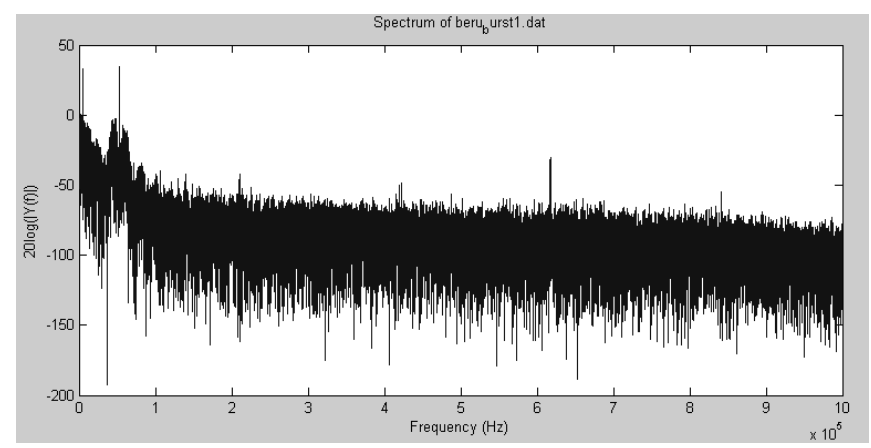

Figure 2 Sensor A measurements in frequency domain

The first 4 bytes are sensor ID value, followed by 1 byte of pressure value $(P)$. Next byte is temperature value $(T)$. Real temperature value in ${ }^{\circ} \mathrm{C}$ is obtained by subtracting 40 from the received unsigned value. After that 2 bytes (CB) with not yet determined purpose (probably status bits) are inserted, which have been always set to a constant value during our measurements. The last 2 bytes are CRC calculated using CRC-16-CCITT standard.

\begin{tabular}{|c|c|c|c|c|}
\hline ID & $\mathrm{P}$ & $\mathrm{T}$ & $\mathrm{CB}$ & $\mathrm{CRC}$ \\
\hline $4 \mathrm{~B}$ & $1 \mathrm{~B}$ & $1 \mathrm{~B}$ & $2 \mathrm{~B}$ & $2 \mathrm{~B}$ \\
\hline
\end{tabular}

Figure 3 Sensor A Message Format

\section{Sensor B}

Sensor B transmits the data after it is activated by a triggering tool or when it is rotated. For triggering it uses a $125 \mathrm{KHz}$ tone 
as low frequency activation signal. The complete activation and transmission process is shown in Figure 4. In this case, the overall communication lasts for $8 \mathrm{sec}$. First, the triggering tool starts with transmitting the wake-up signal. The sensor starts the transmission $3.6 \mathrm{sec}$ after the wake up signal has started.

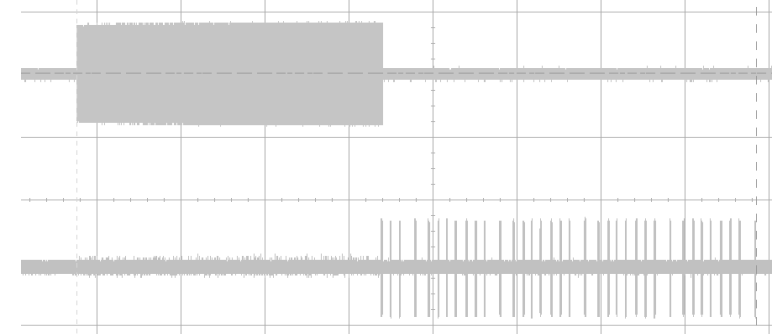

Figure 4 Triggering scenario of Sensor B

After triggering, sensor B responds with 36 identical messages. Each message lasts for $16.5 \mathrm{~ms}$ and the overall session lasts for $4.4 \mathrm{sec}$. Alternatively, when the sensor is rotated, it responds with 6 identical messages (message duration is again $16.5 \mathrm{~ms}$ ).

The detailed view of the transmitted message is shown in Figure 5, and the modulation scheme is On-Off Keying (OOK). The duration of one bit symbol is $240 \mu$ s $(1 / 4.16$ $\mathrm{KHz}$ ). Figure 6 shows the spectrum of the received signal. It can be seen that the RF signal is generated by direct carrier switching.

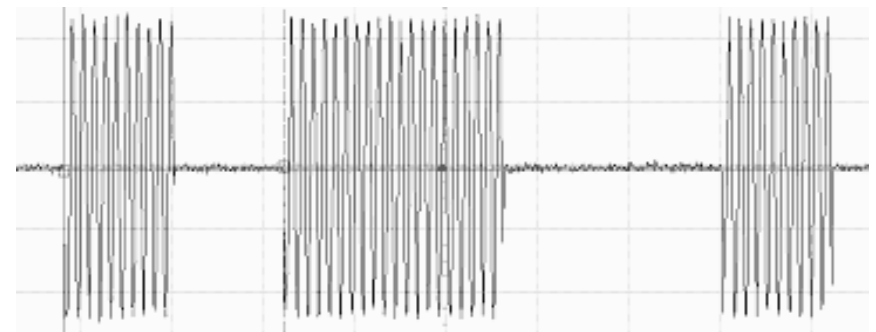

Figure 5 Recorded frame from Sensor $B$ in timing domain

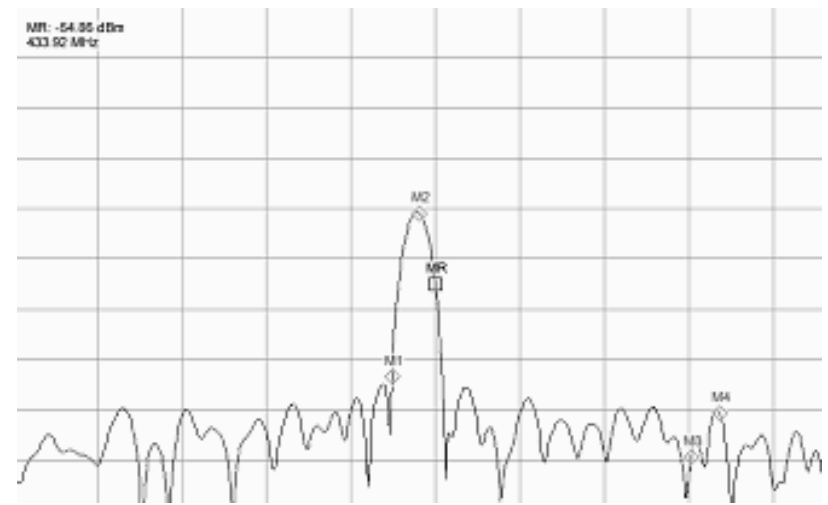

Figure 6 Sensor $B$ measurements in frequency domain

Each message consists of 3 bits +8 bytes ( 3 bits LEARN section +7 data bytes +1 byte CRC) using Manchester code. The preamble consists of a two wide pulses, which is followed by the LEARN section consisting of 3 bits. The first byte after
LEARN section is without determined purpose, which was constant during the measurements (CB). The next 4 bytes are the sensor ID, which is followed by 1 byte pressure value $(\mathrm{P})$ and one byte temperature value $(\mathrm{T})$. The real pressure is obtained by multiplying unsigned received value by 2.5 , while temperature in ${ }^{\circ} \mathrm{C}$ is calculated by subtracting 50 from the received unsigned value. The last byte is CRC according to CRC-8-CCITT standard. LEARN and CB are not used in CRC calculation.

\begin{tabular}{|c|c|c|c|c|c|}
\hline Learn & $\mathrm{CB}$ & $\mathrm{ID}$ & $\mathrm{P}$ & $\mathrm{T}$ & $\mathrm{CRC}$ \\
\hline $3 \mathrm{~b}$ & $1 \mathrm{~B}$ & $4 \mathrm{~B}$ & $1 \mathrm{~B}$ & $1 \mathrm{~B}$ & $1 \mathrm{~B}$ \\
\hline
\end{tabular}

Figure 7 Sensor B Message Format

\section{Sensor $C$}

Sensor $\mathrm{C}$ is activated by a $125 \mathrm{KHz}$ OOK signal, which is shown in Figure 8, and it consists of approximately $15.6 \mathrm{~ms}$ wide pulses, separated with $3 \mathrm{~ms}$ wide pauses.

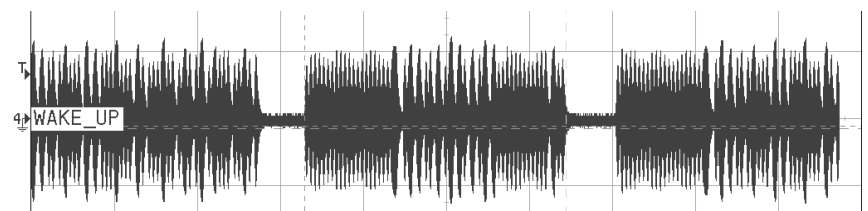

Figure 8 Sensor $\mathrm{C}$ Triggering signal

Sensor $C$ responds to the activation signal within the internal time slot of $27 \mathrm{sec}$ with 4 messages. The first message lasts for approximately $47 \mathrm{~ms}$, and consists of twelve groups made out of 16 pulses, which are probably used for synchronization. Every group of twelve pulses lasts for $1.6 \mathrm{~ms}$. Next three messages carry information on ID, temperature, pressure, etc., which are not identical. Each message lasts for $10.2 \mathrm{~ms}$. A closer view at one of the messages (Figure 9) shows utilized FSK modulation.

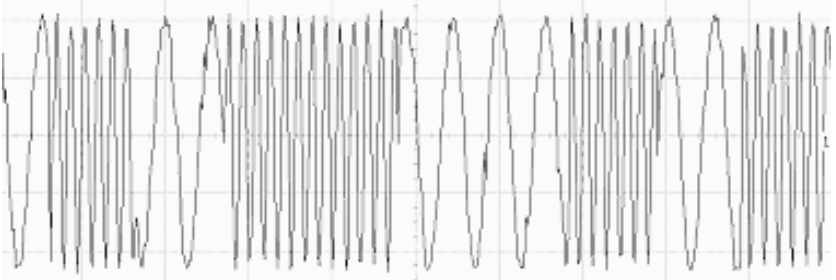

Figure 9 Sensor $\mathrm{C}$ Recorded message in time domain

The first byte is without determined function (CB), which is followed by a 4 byte ID and 1 byte showing the number of the particular message in the session (NM). The next byte is pressure value $(\mathrm{P})$, which is followed by a temperature value (T) and an undetermined byte (CB). The message is ended with 1 checksum (CS) byte, which is simple even parity byte calculated by XOR-ing all data bytes excluding the first byte. The temperature value is calculated by subtracting 50 from received unsigned value. 


\begin{tabular}{|c|c|c|c|c|c|c|}
\hline $\mathrm{CB}$ & $\mathrm{ID}$ & $\mathrm{NM}$ & $\mathrm{P}$ & $\mathrm{T}$ & $\mathrm{CB}$ & $\mathrm{CS}$ \\
\hline 1B & $4 \mathrm{~B}$ & $1 \mathrm{~B}$ & $1 \mathrm{~B}$ & $1 \mathrm{~B}$ & $1 \mathrm{~B}$ & $1 \mathrm{~B}$ \\
\hline
\end{tabular}

Figure 10 Sensor C Message Format

\section{IMPLEMENTATION OF AN EAVESDROPPING SENSOR}

After performing the analysis and decoding of the packets from the different sensors, the next step was to design an evaluation board, which will be able to receive the signal from the TPMS sensors, demodulate, decode, check it and finally send the received data to the PC.

Our sensor platform consists of a power supply, a $\lambda / 4$ antenna, a RF-frontend Chipcon's CC1000, microcontroller MSP430, an ASK demodulation circuit and a RS232 interface to PC. The block diagram of the evaluation board is shown in Figure 11. The utilized RF module is optimized for reception of 433.92 MHz signal. Since this transceiver does not support ASK demodulation, we have used the RSSI (Received Signal Strength Indication) output with additional circuit. The microcontroller is programmed with the software that extracts the useful information from the packets, and communicates with PC. This platform has been successfully utilized in lab environment enabling until now the reception of Sensor A and Sensor B signals.

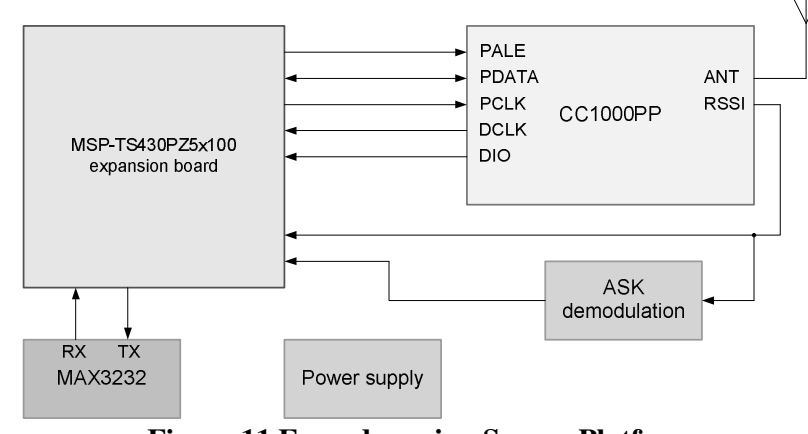

Figure 11 Eavesdropping Sensor Platform

\section{TRAFFIC APPLICATION PERSPECTIVES}

A future low-cost traffic and transportation management system could be realized by using high quality TPMS data of almost any vehicle in the road network. This would augment the current traffic data processes and thus improve the traffic control efficiency, accuracy, reliability and credibility. Such a traffic management and monitoring system based on TPMS would consist of low power sensor nodes (i.e. transceivers), placed near the road. Transceivers should be able to receive signal sent from sensor, extract ID (and if necessary, pressure and temperature) and then transfer the received data to a processing system, e.g. a Traffic Management Center (TMC). There, the data will be processed to the necessary traffic information needed for particular traffic management purposes, e.g. journey times, route flows, origin-destination matrices, etc. to efficiently control traffic by e.g. variable message signs (see Figure 12). For data acquisition two methods can be utilized: wake-up and receive, and eavesdropping.

The first method requires sending of a LF trigger signal in order to activate the sensors. The advantage of this method is that it significantly improves the probability of vehicle identification. One of the key challenges is to ensure that the vehicle is exposed to the trigger signal for a sufficient time to wake-up the sensor. In particular, in the case of fast moving vehicles this could be very difficult having in mind the experienced length of trigger signal, illustrated in the previous section, and extremely low frequency of the trigger signal. These problems could be addressed by application of specific antennas: directional antenna especially designed for this purpose or modified dipole antenna placed along the road. When the sensors are woken up the packets from sensors could be received and sensor IDs identified.

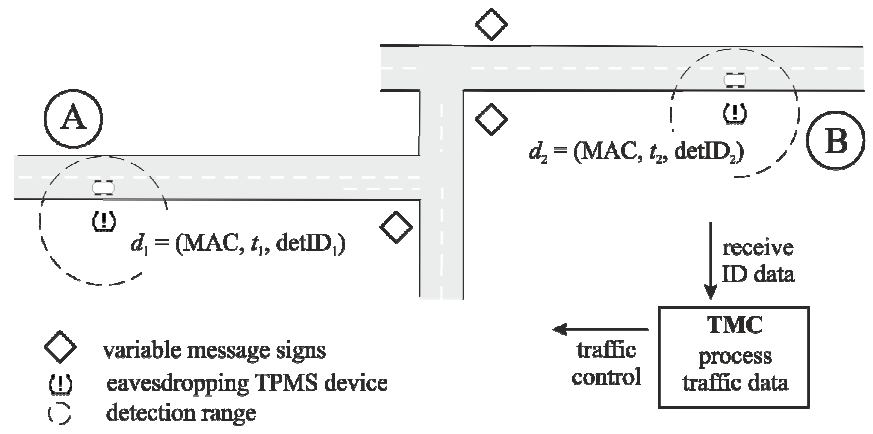

Figure 12 Applying the TPMS system for traffic control

The second method is based just on TPMS signal eavesdropping, by placing receivers along the road. For instance Figure 12, shows two TPMS reader installed along the road to detect the tire IDs (MACs) of the car moving from location A to B. The ID data, which are augmented by the time stamp, the detector IDs (detID) and possibly additional information, are transmitted as data $d_{i}\left(d_{1}\right.$ and $\left.d_{2}\right)$ to the TMC to derive the needed traffic information, e.g. journey times. To ensure capturing of the signal, a set of receivers should be placed near the road assuring to intercept the packet transmission. The number of required sensor nodes depends on the maximum speed of the vehicle $(\mathrm{V})$, the period between the two message transmissions $(\mathrm{T})$ and receiving range of the node (r):

$$
\text { nnodes }=[(\mathrm{V} \cdot \mathrm{T}) / \mathrm{r}]+1
$$

At the moment it is difficult to evaluate the effectiveness of each method, before the field tests are performed, and minimum timing for sensor activation is determined (with the dedicated trigger equipment and without ATEQ VT55). In particular, we need to practically verify the results obtained from the lab. As previously introduced, we have not been able to trigger Sensor A, while Sensor B can be only activated once per $27 \mathrm{sec}$. Having the previous statement in mind, if we assume receiving range of $40 \mathrm{~m} \mathrm{[1]}$, and a speed of $100 \mathrm{~km} / \mathrm{h}$, then more than 400 of such TPMS road-receivers are required to ensure reception of Sensor A. This number is far too big to enable a reasonable use of such a system. As an alternative, one could consider the deployment of a small number of sensors, reducing the probability for successful reception, but still hoping that even reduced number of acquired IDs would be 
sufficient to generate significant statistics of the journey times in the observed area.

The resulting system architecture also depends on the type of the road being monitored (highway, streets, etc.), the number of tracks and the allowed maximum speed. In the case of receiving the signals from all four or even more tires (e.g. trucks), then antennas should be placed on both sides of the road, because of the car body effect [1].

Another important issue is the privacy protection of the measured TPMS data. Although it is much more difficult to disclose the identity, residence, and working place of the traffic participants compared to the ANPR alternative, such malicious act cannot be fully excluded. Thus, an anonymization of the data and a comprehensive data protection concept need to be developed and implemented, which mainly includes hashing algorithms of the IDs. On the other hand, the application of such procedures may limit the usability of TPMS methods for traffic and transportation management purposes, requiring detailed short and long time preserved information about for example commuter and seasonal traffic. Thus, the anonymization needs to be implemented for different time period parameters (minutes, hours, days, weeks, etc.), and in full agreement with the protection laws.

\section{CONCLUSIONS \& FutURE PROSPECTS}

In this paper we analyzed the chances to utilize TPMS systems for traffic and transportation management purposes by a unique identification and reidentification of vehicles. It was demonstrated that with some reverse engineering it is possible to fully analyze and decode the packets sent from TPMS sensors and identify ID address of the sensors. Moreover, it was shown that by using of-the-shelve components simple eavesdropping hardware platform can be implemented and utilized for obtaining the sensor data. Finally, some analysis is performed on how this methodology could be eventually used for traffic management purposes.

The most important open point for future work is to perform the field tests eavesdropping hardware together with TPMS sensors and derive the traffic data in a realistic traffic environment confirming usability of the proposed approach. For this we plan to use DLR test track in Berlin. Furthermore, we will try to improve our eavesdropping hardware platform and its receiving range adding additional LNA and antennas specially optimized for that purpose.

\section{REFERENCES}

[1] I. Rouf, R. Miller, Rob; H. Mustafa, T. Taylor; S. Oh, W. Xu, M. Gruteser, W. Trappe, I. Seskar: Security and Privacy Vulnerabilities of In-Car Wireless Networks: A Tire Pressure Monitoring System Case Study, Proceedings of the 19th USENIX conference on Security, 2010.

[2] S. Velupillai, L. Güvenç, Tire Pressure Monitoring, IEEE Control Systems Magazine, Dec 2007, pp. 22-25.

[3] L. Keilthy, Measuring ANPR System Performance,.Parking Trend International (2008)

[4] RE-BCC7Y Number plate recognition cameras, http://www.dsecctv.com/Prod lettura targhe.htm.
[5] Schnabel, W.; Lohse, D.: Grundlagen der Straßenverkehrstechnik und der Verkehrsplanung. Forschungsgesellschaft für Straßen- und Verkehrswesen e.V., Köln, 1997.

[6] Friedrich, M., Jehlicka, P., Schlaich, J. (2008): Automatic number plate recognition for the observance of travel behavior, 8th International Conference on Survey Methods. in Transport: Harmonisation and Data Comparability, Mai 2008, Annecy, France.

[7] Autorenkollektiv FGSV (2010): „Hinweise zur kurzzeitigen automatischen Erfassung von Daten des Straßenverkehrs“, Forschungsgesellschaft für Straßen- und Verkehrswesen (Hrsg.), Köln, 2010

[8] Kathawala, Y. A. and Tueck, B : "The use of RFID for traffic management". In International Journal of Technology, Policy and Management, Inderscience Enterprises Ltd., Volume 8, Number 2/2008, pp. 111-125, 2008

[9] Virtanen, J.: "Mobile phones as probes in travel time monitoring - The general description of pilot implementation“. Finish Road Administration, Helsinki, Finnland, 2002.

[10] European Patent, Monitoring a Mobile Device, patent number EP000002232456A4

[11] European Patent, Estimation of Travel Times Using Bluetooth , patent number EP000002320403A3

[12] Hsieh, J.-W.; Yu, S.-H.; Chen, Y.-S.: Morphology-based license plate detection in images of differently illuminated cars. In Journal of Electronic Imaging, Vol. 11, pp. 507-516, 2002.

[13] Hsieh, C.-T.; Juan, Y.-S.; Hung, K.-M.: Multiple License Plate Detection for Complex Background. In Proceedings of the 19th International Conference on Advanced Information Networking and Applications, Vol. 2, pp. 389-392, 2005.

[14] Matas, J.; Zimmermann, K.: Unconstrained License Plate and Text Localization and Recognition. In 8th International IEEE Conference on Intelligent Transportation Systems, pp. 572- 577, Heidelberg, Germany, September 2005.

[15] Leich, A.: Ein Beitrag zur Realisierung der videobasierten weiträumigen Verkehrsbeobachtung. Ph.D. thesis, Dresden University of Technology Dresden, 2006.

[16] Schulz, J., Walayat, Y: Towards a traffic measuring surveillance system utilizing tire pressure monitoring systems. In 4th Nearctis Workshop, Ifsttar, Lyon, 1Oth June 2011

[17] Schulz, J, Junghans, M.: Deployment of Tire Pressure Monitoring Systems for Traffic Monitoring and Safety Purposes. In $18^{\text {th }}$ World Congress on Intelligent Transportation Systems, $16^{\text {th }}-20^{\text {th }}$ October 2011, Orlando, USA

[18] Behrisch, M.; Bieker, L.; Erdmann, J.; Krajzewicz, D.: SUMO Simulation of Urban Mobility - An Overview. In SIMUL 2011 : The Third International Conference on Advances in System Simulation, 23th-29 ${ }^{\text {th }}$ November, Barcelona, Spain.

[19] PTV Planung und Transport AG: URL: www.vissim.de/ (30.04.2012)

[20] Spangler, M.; Leonhardt, A.; Busch, F.; Carstensen, C.; Zeh, T... Deriving travel times in road networks using Bluetooh-based vehicle reidentification: Experiences from Northern Bavaria, FOVUS - Networks for Mobility, ISBN: 978-3-00-031958-7, 2010.

[21] Weinzerl, J. (2010). Verkehrsablauf erkennen durch intelligente Sensoren - auf Autobahnen und in urbanen Gebieten, Tagungsband HEUREKA '10, Editor Forschungsgesellschaft für Straßen- und Verkehrswesen. 